\title{
Spectroscopic investigation of excitons, photocarriers, and bias-induced carriers in regioregular poly(3-alkylthiophene)
}

Katsuichi Kanemoto, Motoaki Yasui, Tatsuya Higuchi, Daisuke Kosumi, Ichiro Akai, Tsutomu Karasawa, Hideki Hashimoto

\begin{tabular}{|c|l|}
\hline Citation & Physical Review B, 83(20); 205203 \\
\hline Issue Date & 2011-05-15 \\
\hline Type & Journal Article \\
\hline Textversion & Publisher \\
\hline \multirow{3}{*}{ Rights } & $\begin{array}{l}\text { CAmerican Physical Society. This article may be downloaded for personal use } \\
\text { only. Any other use requires prior permission of the author and American Physical } \\
\text { Society. } \\
\text { The following article appeared in Physical Review B Vol.83, Iss.20 and may be } \\
\text { found at https://doi.org/10.1103/PhysRevB.83.205203 }\end{array}$ \\
\hline DOI & \begin{tabular}{l}
$10.1103 /$ PhysRevB.83.205203 \\
\hline
\end{tabular}
\end{tabular}

\author{
Self-Archiving by Author(s) \\ Placed on: Osaka City University
}

Katsuichi Kanemoto. et al. (2011). Spectroscopic investigation of excitons, photocarriers, and bias-induced carriers in regioregular poly(3-alkylthiophene). Physical Review B. 83, 205203. https://doi.org/10.1103/PhysRevB.83.205203 


\title{
Spectroscopic investigation of excitons, photocarriers, and bias-induced carriers in regioregular poly(3-alkylthiophene)
}

\author{
Katsuichi Kanemoto, ${ }^{1, *}$ Motoaki Yasui, ${ }^{1}$ Tatsuya Higuchi, ${ }^{1}$ Daisuke Kosumi, ${ }^{1}$ Ichiro Akai, ${ }^{2}$ \\ Tsutomu Karasawa, ${ }^{1}$ and Hideki Hashimoto ${ }^{1,3}$ \\ ${ }^{1}$ CREST/JST and Department of Physics, Osaka City University, 3-3-138 Sugimoto, Sumiyoshi-ku, Osaka 558-8585, Japan \\ ${ }^{2}$ Shock Wave and Condensed Matter Research Center, Kumamoto University, 39-1 Kurokami 2, Kumamoto 860-8585, Japan \\ ${ }^{3}$ The OCU Advanced Research Institute for Natural Science and Technology (OCARINA), Osaka City University, Osaka 558-8585, Japan
}

(Received 2 August 2010; revised manuscript received 11 February 2011; published 18 May 2011)

\begin{abstract}
Excitons, photocarriers, and bias-induced carriers in regioregular poly(3-alkylthiophene) (P3AT) films were investigated using a variety of spectroscopic techniques, such as continuous-wave- (cw-) and femtosecond (fs-) transient photoinduced absorption (PIA) and device modulation (DM) spectroscopies. Comparison between the cw PIA and DM spectra of the poly(3-hexylthiophene) (P3HT) film reveals that photocarriers generated by cw photoexcitation are dominated by localized polarons, whereas charge injection primarily creates delocalized polarons. The photoexcitation intensity dependence of the cw PIA signals demonstrates that both localized and delocalized photogenerated polarons obey a bimolecular recombination process under $\mathrm{cw}$ photoexcitation. The proportion of polarons generated by $\mathrm{cw}$ photoexcitation in the P3HT film is estimated to be $1 /\left(1+0.137 I^{1 / 2}\right)$ for $I\left(\mathrm{~mW} / \mathrm{cm}^{2}\right)$ by comparison of the spectral intensities between the DM and cw PIA spectra. DM spectroscopy reveals that both localized and delocalized polarons rapidly increase at a rise point of the bias current. The $\mathrm{cW}$ PIA bleaching spectra of P3AT films are shown to vary with the length of the alkyl sidechains of P3AT and be almost reproduced using a single Huang-Rhys factor. The Huang-Rhys factors obtained reveal a trend that the electron-phonon couplings of final exciton migration sites become strong with the increase in the interlamella distance in the P3AT film, which indicates that interlamella couplings affect the electron-phonon couplings of the migration sites located at two-dimensional lamellas.
\end{abstract}

DOI: 10.1103/PhysRevB.83.205203

PACS number(s): 78.66.Qn, 73.61.Ph, 78.47.jb, 71.35.Cc

\section{INTRODUCTION}

Conjugated polymers have a large range of applications, due to their wide variety of optical and electronic properties. Among conjugated polymers, head-to-tail-coupled, regioregular (RR) poly(3-alkylthiophenes) (P3ATs) with alkyl sidechains are regarded as being somewhat unique, because they form solid films that have self-organized microcrystalline domains. $^{1-3}$ The formation of such microcrystalline domains was demonstrated to be effective for enhancement of charge carrier mobility in the field-effect transistor (FET) of a representative P3AT, poly(3-hexylthiophene) (P3HT). ${ }^{3}$ RR $\mathrm{P} 3 \mathrm{HT}$ has thus been considered to be one of the most promising polymers for FET applications. In addition, RR P3AT can be regarded as a prototype of aggregate phases that are partly present in conventional disordered amorphous polymer films. Therefore, the RR P3AT film is also useful as a system allowing for an investigation of the device physics and photophysics of such polymer aggregate phases.

The photophysics of the RR P3AT film has been investigated using spectroscopic techniques. Recently, many studies were reported concerning the photoexcitation dynamics of P3AT films determined by transient absorption techniques, ${ }^{4-12}$ and the understanding of the photophysics in the early stage of photoexcitation has been developed. By contrast, there have been only a few reports of the photophysics in the slow time regime of the RR P3AT film. ${ }^{4,13}$ In this paper, the photophysics of the RR P3AT film under continuouswave (cw) photoexcitation is addressed. The photophysics is important not only for discussing the photoexcitation dynamics in the slow time regime but also for enhancing the performance of the RR P3AT/fullerene composite solar cells that are designed for use under cw photoirradiation. ${ }^{16,17}$ We here adopt a cw photoinduced absorption (PIA) technique, which has been shown to be effective for investigating the slow-regime photoexcitation dynamics of conjugated polymers ${ }^{4,13-15,18-23}$ and must also be effective for directly accessing the photophysics under $\mathrm{cw}$ photoirradiation. In the slow time regime, clarifying the behaviors of photogenerated polarons is the key to revealing the dynamics of the RR P3AT film since triplet excitons were shown not to be generated in the RR P3AT film by magnetic resonance techniques. ${ }^{4}$ We thus focus on the photophysics of the photogenerated polarons under $\mathrm{cw}$ photoexcitation. In addition, we also examine the spectral features of polaron carriers induced by applying a bias in the RR P3HT diode using device modulation (DM) spectroscopy that observes optical probe signals from bias-induced carriers. ${ }^{9,24-26}$ Through a comparison of the spectral features from cw PIA and DM measurements, we attempt to reveal the difference in the electronic structures of photogenerated and bias-injected polarons.

In addition, the photophysics of photogenerated singlet excitons in the P3AT films is also examined on the basis of bleaching signals of cw-transient and femtosecond- (fs-) transient PIA measurements. The bleaching signals enable us to observe the absorption spectrum of components consumed by photoexcitation. We have recently applied bleaching spectroscopy to the RR P3HT film and demonstrated that the film has several morphological components, and the coexistence of the components can determine the conducting and optical properties of polymer films. ${ }^{9}$ The presence of such different components has not been considered in discussing the photophysics and device physics in the RR P3AT film, 


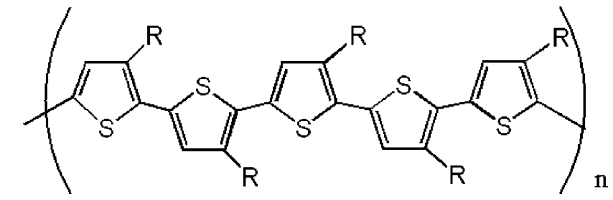

FIG. 1. Chemical structure of the repeat unit of RR P3AT: $\mathrm{R}=\mathrm{C}_{4} \mathrm{H}_{9}$ for RR P3BT, $\mathrm{R}=\mathrm{C}_{6} \mathrm{H}_{13}$ for RR P3HT, $\mathrm{R}=\mathrm{C}_{8} \mathrm{H}_{17}$ for RR P3OT, and $\mathrm{R}=\mathrm{C}_{12} \mathrm{H}_{25}$ for RR P3DDT.

excluding our previous study. ${ }^{9}$ We attempt to reveal the behavior of photocarriers, bias-induced carriers, and excitons by considering the coexistence of different components in explaining the results of spectroscopic measurements. This attempt will provide new insight into understanding the photophysics and device physics in RR P3AT films.

\section{EXPERIMENTAL}

RR P3ATs used in this study were RR poly(3butylthiophene) (P3BT), RR P3HT, RR poly(3octylthiophene) (P3OT), and RR poly(3-dodecylthiophene) (P3DDT) (Fig. 1), which were purchased from Aldrich and used as received. Optical measurements were performed on RR P3AT films. The RR P3AT films were fabricated by spin-casting a chlorobenzene solution of RR P3AT at a concentration of $10 \mathrm{mg} / \mathrm{mL}$. The thickness of the RR P3HT films was approximately $80 \mathrm{~nm}$.

For the cw PIA measurements, excitation was provided by an argon-ion laser at $488 \mathrm{~nm}$ (typically $\sim 50 \mathrm{~mW}$ ) modulated with an acoustic-optic modulator at $700 \mathrm{~Hz}$. The probe beam for the measurement was produced using a tungsten/halogen lamp. The $\mathrm{cw}$ PIA signals were measured using a phasesensitive lock-in technique with a monochromator and a $\mathrm{Si}$ photodiode. Photoluminescence (PL) signals were eliminated by subtracting the spectrum measured without the probe beam. The photoexcitation intensity dependence of the $\mathrm{cW}$ PIA signals was carefully measured in the range of photoexcitation that does not lead to serious heating and degradation of the sample. The laser system for transient PIA measurements employed a Ti:sapphire laser (Spectra Physics, Hurricane-X) with a repetition rate of $1 \mathrm{kHz}$ at $780 \mathrm{~nm}(\sim 100$-fs pulse duration). A portion of the beam from the Ti:sapphire amplifier was used to pump an optical parametric amplifier (OPA; Spectra Physics, OPA-800C). Frequency-tunable visible pump pulses were produced by sum-frequency mixing the OPA signal with the residual of the 780 -nm fundamental beam in a 1-mm-thick type II $\beta$ - $\mathrm{BaB}_{2} \mathrm{O}_{4}$ crystal. The pump pulse was tuned to $480 \mathrm{~nm}$ and used for excitation $(50 \mathrm{~nJ} /$ pulse). A white-light continuum was produced in a sapphire plate using a portion of the amplified 780-nm beam and was used as probe pulses of variable wavelengths. The probe pulses after the sample were dispersed with a spectrograph and imaged by a 1024-pixel linear photodiode array designed for fast readout and low noise. ${ }^{27-29}$

DM spectroscopy was performed for a diode device made from RR P3HT. The device consisted of four layers: an indium-tin-oxide (ITO) coated glass substrate, a hole-transporting poly(3,4-ethylenedioxythiophene)poly(styrenesulfonate) (PEDOT-PSS) layer, the RR P3HT film, and a semitransparent aluminum electrode deposited by vacuum evaporation. A DM spectrum was obtained by applying dc and squared ac voltages $\left(V_{\mathrm{DC}}=0 \mathrm{~V}\right.$ and $V_{\mathrm{AC}}$ $=1 \mathrm{~V}$, respectively) to the diode device and detecting the modulated signal of the transmitted light synchronized with the frequency of the ac voltage $(700 \mathrm{~Hz})$. Electroabsorption (EA) measurements were performed using the coplanar electrode configuration against polymer films with an electrode spacing of $50 \mu \mathrm{m}$. The same probe system as that used for the cw PIA measurement was used for detection. All optical measurements were performed at room temperature in an inert atmosphere.

\section{RESULTS AND DISCUSSION}

\section{A. Spectroscopy of polaron carriers}

Figures 2(a), 2(b), and 2(c) show the absorption, cw PIA (photoexcitation intensity is $2 \times 10^{3} \mathrm{~mW} / \mathrm{cm}^{2}$ ), and DM spectra of the RR P3HT film, respectively. Both of the cw PIA and DM spectra exhibit clear absorption and bleaching signals induced by $\mathrm{cw}$ photoexcitation and bias impression, respectively. The absorption peaks of the cw PIA spectrum at 1.25 and $1.82 \mathrm{eV}$ are assigned to localized polarons (LPs) and delocalized polarons (DPs), respectively, according to previous reports that assigned the peaks using magnetic resonance techniques. ${ }^{4,14}$ The cw PIA spectrum does not contain a contribution from the absorption band of trapped interchain singlet excitons, which the previous reports observed around 1.05 $-1.10 \mathrm{eV}$ at low temperatures. ${ }^{4,14}$ The lack of the exciton band would be due to the difference of measurement temperature since we observed the band only at low temperatures.

Absorption peaks at 1.30 and $1.83 \mathrm{eV}$ in the DM spectrum are also assigned as the LP and DP bands, respectively, similar to the PIA spectrum. In contrast, the intensity ratio of the LP to DP band is obviously different between the cw

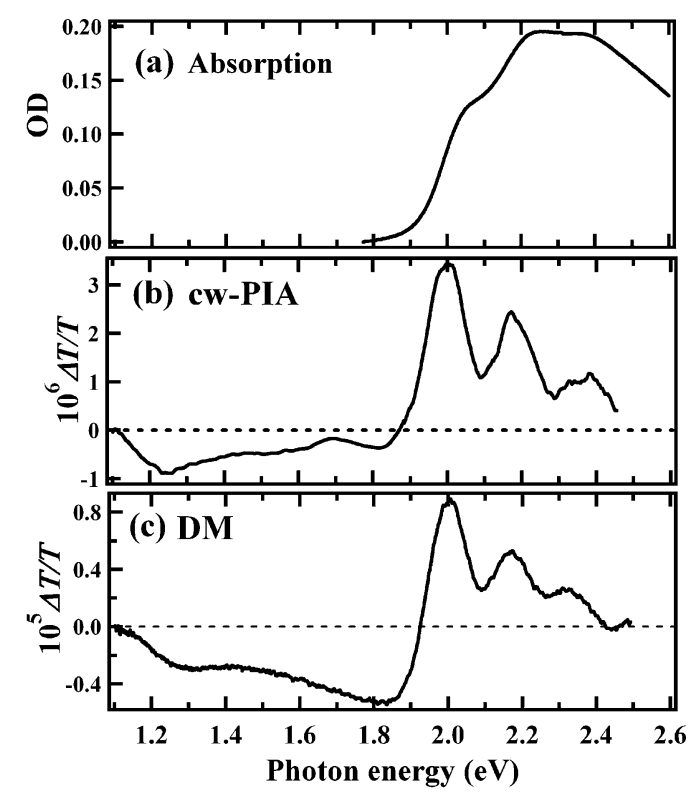

FIG. 2. (a) Steady-state absorption, (b) cw PIA and (c) DM spectra for the RR P3HT film. Photoexcitation intensity for the $\mathrm{cw}$ PIA measurement was $2 \times 10^{3} \mathrm{~mW} / \mathrm{cm}^{2}$. 
PIA and DM spectra; the LP band is greater in the cw PIA spectrum and the DP band is greater in the DM spectrum. This indicates that the electronic states of polaron carriers generated by photoexcitation and charge injection are different in the P3HT film. Photocarriers generated by cw photoexcitation are dominated by localized polarons, whereas charge injection primarily creates delocalized polarons.

The relative change in the optical transmission $T$ induced by photoirradiation is given by $\Delta T / T \approx-d \Delta \alpha$, where $d$ is the sample thickness and $\Delta \alpha$ is the photoinduced absorption. This equation relates the modulated signal $\Delta T$ to the density of photoexcitations $N$. The response of $N$ to the photoirradiation intensity $I$ is described by the following rate equations,

$$
\frac{d N}{d t}=\eta g I-\beta N
$$

for a monomolecular recombination process, and

$$
\frac{d N}{d t}=\eta g I-\gamma N^{2}
$$

for a bimolecular recombination process, where $\eta$ is the photoexcitation quantum efficiency, $g$ is the generation constant, and $\beta$ and $\gamma$ are the monomolecular and bimolecular recombination constants, respectively. ${ }^{19,20} N$ in cw photoirradiation is obtained by a steady-state solution of Eq. (1) or (2). It follows from the solution that $\Delta T / T(\propto N)$ is proportional to $I$ for the monomolecular process and to $I^{1 / 2}$ for the bimolecular process. In the case of the bimolecular process, a steady-state condition in cw PIA is obtained when $\omega \tau_{\gamma} \ll 1$, where $\omega$ is the modulation angular frequency of photoexcitation and $\tau_{\gamma}$ is the bimolecular lifetime obtained by $(\eta g I \gamma)^{-1 / 2} .22 \mathrm{We}$ can determine from the photoexcitation intensity dependence of the cw PIA signals whether the monomolecular or the bimolecular process is dominant.

Figure 3 shows the photoexcitation intensity dependence of the LP $(1.25-\mathrm{eV})$ and DP band $(1.82-\mathrm{eV})$ intensities of the $\mathrm{cw}$ PIA spectrum for the RR P3HT film. The intensities of both bands increase nonlinearly with the photoexcitation intensity. The intensity dependence was thus fitted using the square root of the intensity $\left(I^{1 / 2}\right)$. The best-fit result is shown in Fig. 3 and demonstrates that the intensity dependence of both bands is almost reproduced by the $I^{1 / 2}$ function. It is thus concluded that both LP and DP obey a bimolecular recombination process under $\mathrm{cw}$ photoexcitation. However, the intensity dependence of both LP and DP bands seems somewhat linear below $300 \mathrm{~mW} / \mathrm{cm}^{2}$. Therefore, the bimolecular recombination process may occur above $300 \mathrm{~mW} / \mathrm{cm}^{2}$. Figure 3 also shows the photoexcitation dependence of the PL intensity for the RR P3HT film. The PL intensity increases linearly with the photoexcitation intensity. Therefore, singlet excitons responsible for PL in the RR P3HT film obey a monomolecular recombination process under $\mathrm{cw}$ photoexcitation. It is thus suggested that the proportion of singlet excitons to polaron carriers increases with the increase of the photoexcitation intensity.

Figure 4 shows the voltage dependence of the DM signal intensity for the RR P3HT film detected at the LP $(1.30-\mathrm{eV})$ and DP $(1.83-\mathrm{eV})$ bands. This voltage dependence was measured by modulating squared ac voltages between $0 \mathrm{~V}$ and the indicated voltage corresponding to applying $x / 2 \pm$

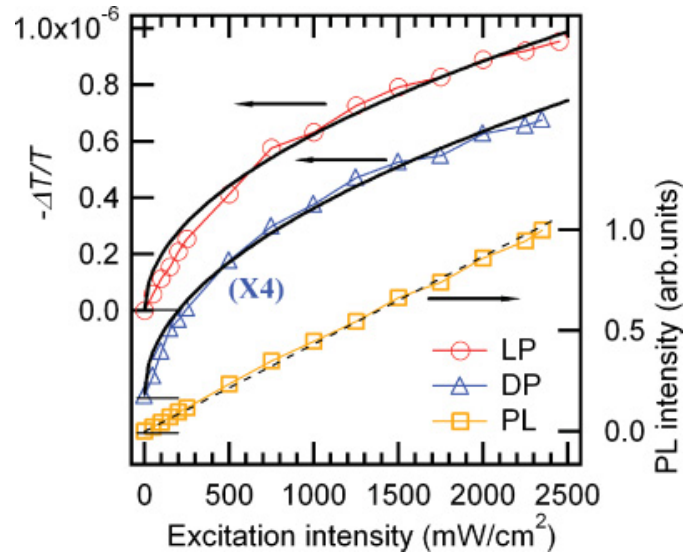

FIG. 3. (Color online) Photoexcitation intensity $(I)$ dependencies of the LP signal at $1.25 \mathrm{eV}$ and DP signal at $1.82 \mathrm{eV}$ for the cw PIA spectrum and PL intensity for the RR P3HT film. The three horizontal solid lines are the baselines for the three dependencies. Solid curves for the LP and DP signals are the best-fit results obtained using a function of the square root of the intensity $\left(I^{1 / 2}\right)$, and the dashed line for the PL signals is the best-fit result obtained using a linear dependence of $I$.

$x / 2 \mathrm{~V}$ for a signal at $x \mathrm{~V}$. The result demonstrates that both the LP and DP bands increase linearly with voltage below $1 \mathrm{~V}$, and then exhibit a more rapid increase above $1 \mathrm{~V}$. This turning point at $1 \mathrm{~V}$ is consistent with the current-voltage characteristics, which showed behavior typical of the space-charge-limited current (SCLC). ${ }^{9}$ Below $1 \mathrm{~V}$, the current of the P3HT diode increased linearly with voltage, typical behavior for ohmic electrical conduction. ${ }^{9}$ In ohmic conduction, the number of carriers is assumed to remain constant as the voltage increases. Therefore, the DM signals below $1 \mathrm{~V}$ are not obtained by carriers responsible for the ohmic conduction. We consider that the immobile or trapped carriers generated by applied bias may contribute to the DM signals in this voltage region.

By contrast, the increase of the DM signals above $1 \mathrm{~V}$ is expected to be due to mobile polarons, since it is consistent with the rise of the SCLC behavior. The rises of the current and the DM signals above $1 \mathrm{~V}$ were fitted using a power function of voltage $\left(V^{n}\right)$ to examine a relation between them. The best-fit results were $n=2.31,1.20$, and 1.23 for the current, the LP bands and the DP bands in the DM signals, respectively; the results of fit for the LP and DP signals are shown in Fig. 4. The SCLC conduction typically increases depending on the square of voltage, which is given by linear increases of carrier number and electric field against a voltage increase. ${ }^{30}$ The obtained $n$ value somewhat larger than 2 in the current may be due to a distribution of energy levels in mobile carriers. In this case, the rise of the DM signals should increase with a $V$ dependence of $n \approx 1.3$, which is somewhat larger than the obtained values in the LP and DP bands. We consider that this discrepancy would arise from a change in the carrier mobility depending on voltage that contributes only to the current.

Bleaching signals of a cw PIA spectrum are given by an absorption spectrum of components consumed by $\mathrm{cw}$ photoexcitation. A bleaching spectrum thus provides information on the location of photoexcited species. In the case of the RR P3HT film, the bleaching spectrum of a PIA spectrum 


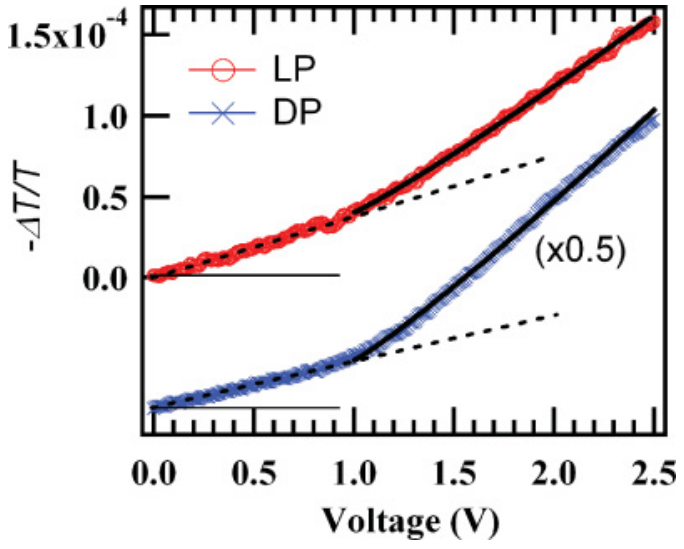

FIG. 4. (Color online) Voltage dependence of DM signal intensity for the RR P3HT film detected at the LP $(1.30 \mathrm{eV})$ and DP (1.83 $\mathrm{eV}$ ) bands measured by modulating squared $\mathrm{AC}$ voltages between indicated voltage and $0 \mathrm{~V}$. The two horizontal solid lines indicate the baselines for the two dependences. The dashed lines are the results of the best fit obtained using a linear dependence of voltage below $1 \mathrm{~V}$. The solid lines are the results of the best fit for the voltage dependence above $1 \mathrm{~V}$ obtained using the sum of a power function of voltage $\left(V^{n}\right)$ and the linear voltage dependence.

primarily reflects the location of singlet excitons. ${ }^{9}$ In particular, the bleaching spectrum in the cw PIA measurement shown in Fig. 2(b) is almost determined by the final migration sites of excitons. ${ }^{9}$ Therefore, the difference between the cw PIA bleaching and steady-state absorption spectra in Fig. 2(a) indicates that the excitons are finally localized at specific sites in the film. Bleaching signals of a DM spectrum correspond to the absorption spectrum of neutral components converted into carriers by charge injection, and the bleaching spectrum provides information on the location of injected carriers. The bleaching spectrum of the DM spectrum in Fig. 2(c) is similar to that for the cw PIA spectrum, which suggests that injected polaron carriers under the bias range are located at similar sites to which the excitons finally migrate.

By contrast, comparison of the ratio of the bleaching intensity with the polaron band intensity for the $\mathrm{cW}$ PIA and DM spectra indicates that the relative polaron band intensity is weaker in the cw PIA spectrum. This suggests that the proportion of polarons generated from photoexcitation is low. We now assume that DM signals from excitons are negligibly small, and hence that the areal ratio of the polaron band signals to the bleaching signals in the DM spectrum corresponds to a case where only polaron carriers are generated by bias impression. It is also noted that the contribution of EA signals to the DM spectrum is negligible. ${ }^{9}$ An estimate of the proportion of polarons from photoexcitation is then made for the cw PIA spectrum based on these assumptions.

For simplicity, we further assume that the oscillator strength of the LP and DP bands is the same and that the absorption component of the observed cw PIA spectrum is given only by polaron carriers including mobile and trapped carriers. Overlapped signals around the crossover point between bleaching and absorption components are also neglected. The integrals of the bleaching signals in the cw PIA and DM spectra were calculated below $2.3 \mathrm{eV}$. The integral ratio of the absorption component to the bleaching component was calculated to be 0.46 for the cw PIA spectrum and 1.6 for the DM spectrum. In cw photoexcitation, negative polarons are simultaneously generated with positive polarons, and we assume that both polarons yield the same integral intensity in the spectral range, based on recent theoretical work on thiophene oligomers. ${ }^{31}$ It is then concluded that the proportion of positive or negative polarons in the photoexcitations is 0.14 under a photoexcitation intensity of $2 \times 10^{3} \mathrm{~mW} / \mathrm{cm}^{2}$. This small value is consistent with the low photoexcitation quantum efficiency of polarons determined previously using transient absorption measurements. ${ }^{5,8}$ The remainder of the photoexcitations (0.86 in proportion) should be mostly excitons. Whereas the cw PIA signals of LP and DP bands increase with $I^{1 / 2}$ under $I>300 \mathrm{~mW} / \mathrm{cm}^{2}$, the PL increases linearly with $I$, as shown in Fig. 3. This indicates that the proportion of polarons increases with decreasing $I$ because of a reduction in the recombination of polarons. We now assume that the photoexcited species excluding the polarons are all excitons, and PL simply increases with the number of photogenerated excitons. The $I$ dependence of the proportion of polarons from photoexcitation can then be estimated using the relationships of $I$ dependence with the cw PIA and PL signals. Calculations conclude the proportion of polarons to be $1 /\left(1+0.137 I^{1 / 2}\right)$ for the photoexcitation intensity $I\left(\mathrm{~mW} / \mathrm{cm}^{2}\right)$ under $I$ $>300 \mathrm{~mW} / \mathrm{cm}^{2}$. Although this relation was derived under several assumptions, it enables us to estimate the polaron proportion in the RR P3HT films over a wide range of $\mathrm{cW}$ photoexcitation intensity as long as the condition $\omega \tau_{\gamma} \ll 1$ is satisfied.

\section{B. Final migration sites in a series of P3AT films}

The excitons of the P3HT film have been shown to finally arrive at specific minor sites in the film. Elucidating the nature of the final arriving sites is important because they can determine photophysical properties under $\mathrm{cw}$ photoirradiation. It is known that RR P3AT forms solid films with microcrystalline lamella structures and that the interlamella distance varies with the length of the alkyl substituents: $12.63 \AA$ (P3BT), $16.36 \AA$ (P3HT), $20.10 \AA$ (P3OT) and $27.19 \AA$ (P3DDT). ${ }^{2}$ We can thus investigate whether such final migration sites depend on the interlamella distance from the bleaching spectra of a series of P3AT films. Figure 5 shows the cw PIA bleaching spectra of the RR P3BT, RR P3HT, RR P3OT, and RR P3DDT films. The line shapes of the bleaching spectra change with the length of the alkyl sidechains. In addition, Fig. 6 presents the transient PIA spectra for the RR P3OT film recorded at 10, 50, 100, and $300 \mathrm{ps}$ after photoexcitation. The spectra demonstrate that the spectral line shape varies with time, similar to the case of RR P3HT reported previously. ${ }^{9}$

It was recently reported that the bleaching components of transient PIA signals for a P3HT film are due to a contribution of EA from ground-state chromophores, due to the local field of charge pairs. ${ }^{11}$ Therefore, we discuss the contribution of EA signals to the PIA spectra before considering the origin of the observed bleaching spectra. Figure 7 shows comparison of EA spectra for the RR P3HT and RR P3DDT films. The EA spectra exhibit a line shape similar to that of previous reports ${ }^{14,32,33}$ and 


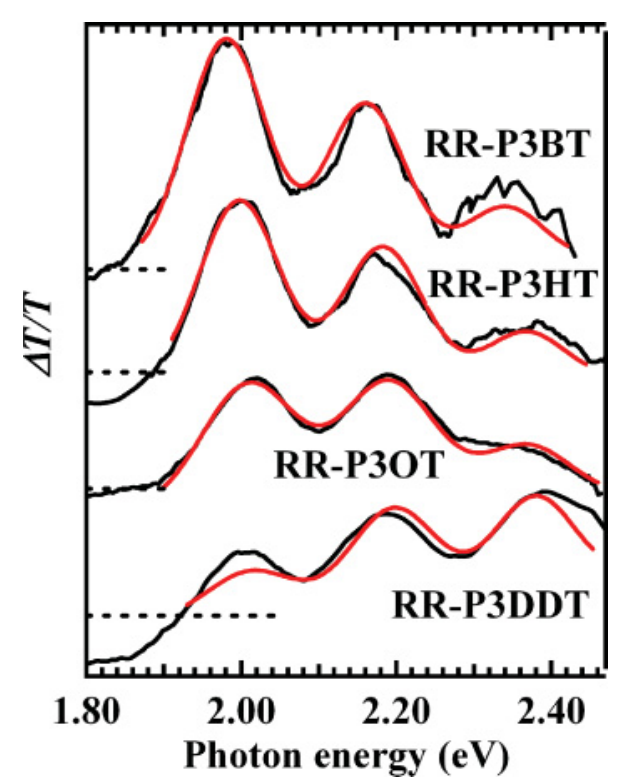

FIG. 5. (Color Online) Bleaching spectra obtained from cw PIA measurements for the RR P3BT, RR P3HT, RR P3OT, and RR P3DDT films. The red solid curve for each spectrum is the best-fit result of simulation obtained using a single Huang-Rhys factor.

demonstrate that the line shape is almost independent of the alkyl sidechain length. The EA spectrum of the RR P3HT film is somewhat similar to the cw PIA bleaching spectrum shown in Fig. 2(b). However, the EA and cw PIA bleaching spectra of the RR P3DDT film are obviously different. Furthermore, the observed bleaching spectra give only positive signals, whereas the sign of the EA signals changes at around $2.2 \mathrm{eV}$. These spectral features confirm that the cw PIA bleaching spectra in Fig. 5 are not given by the EA signals. This also leads to a conclusion that the transient bleaching spectra are also not due to EA components, because the previously reported bleaching spectra of the transient PIA for the RR P3HT film ${ }^{9}$ consist of a spectral component similar to the cw PIA bleaching and

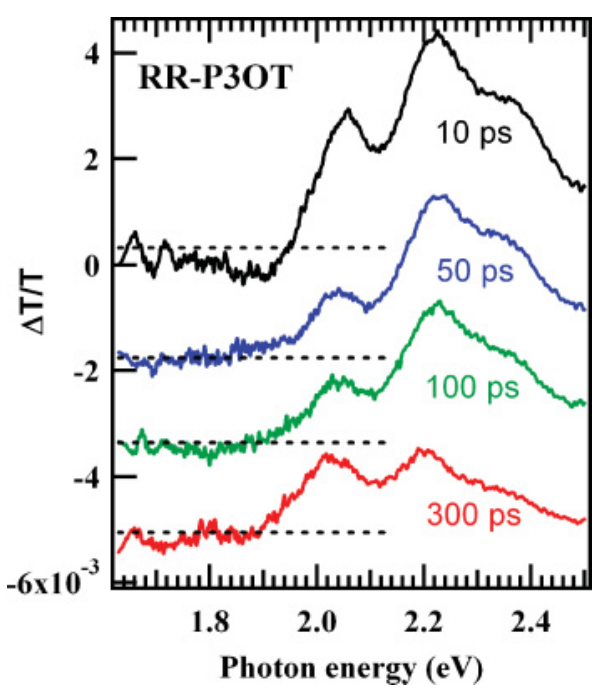

FIG. 6. (Color online) Transient PIA spectra for the RR P3OT film recorded at 10, 50,100, and $300 \mathrm{ps}$. The horizontal dashed lines indicate the baselines for each spectrum.

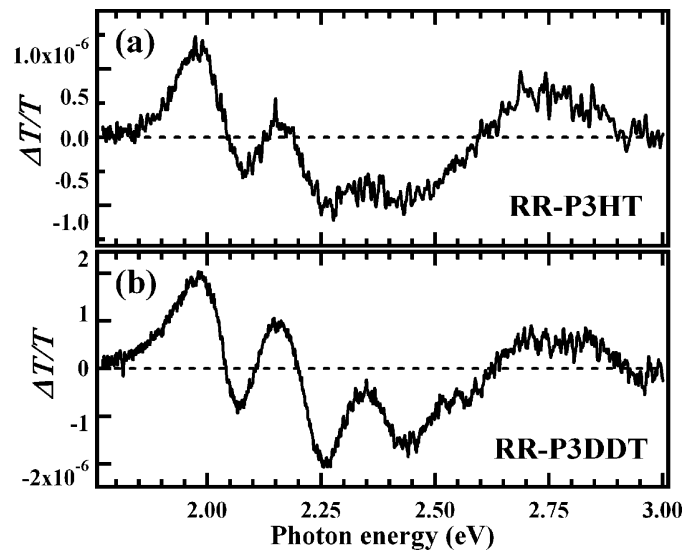

FIG. 7. Electroabsorption spectra for the (a) RR P3HT and (b) RR P3DDT films.

a component observed in the early stage of photoexcitation that is not similar to the EA spectrum. The observed temporal bleaching shift of P3OT in Fig. 6 is thus attributed to the migration of excitons. The bleaching spectrum at $10 \mathrm{ps}$ has a different line shape from that of its cw PIA bleaching spectrum and is similar to the transient bleaching spectra of RR P3HT at 0 and 10 ps reported previously. ${ }^{9}$ This indicates that similar morphological components are consumed in the early stage of photoexcitation for the P3HT and P3OT films, but their final migration sites are different.

Whether a spectrum is composed of a single component or several can be determined by fitting the spectrum with a Franck-Condon (FC) model using a single Huang-Rhys (HR) factor. The FC model describes the relative intensity of the phonon replica as being given by

$$
I(\omega) \propto \sum_{m} \frac{S^{m} \exp (-S)}{m !} \Gamma\left(\omega-\omega_{0}-m \omega_{p}\right),
$$

where $S$ is the HR factor and $\Gamma$ is the line-shape operator. The terms $\omega_{0}$ and $\omega_{p}$ are the 0-0 transition frequency and the phonon frequency, respectively. FC analyses were performed using a single HR factor for the bleaching spectra in Fig. 5. In the analyses, we treated only a single-phonon mode, causing apparent splittings. The results of the fit are shown in Fig. 5 and the parameters obtained from the analyses are given in Table I. The fitted curves demonstrate that the cw PIA spectra of the P3AT films are almost reproduced by a simple FC progression using a single HR factor, which indicates that each bleaching spectrum primarily consists of a single component.

TABLE I. Parameters for the best-fit simulations obtained using a single (HR) factor shown in Fig. 4; the Gaussian full width at half-maximum (FWHM), the 0-0 transition energy $\left(E_{0}\right)$, and the average phonon energy $\left(E_{p}\right)$.

\begin{tabular}{lcccc}
\hline \hline Sample & HR factor & FWHM (meV) & $E_{0}(\mathrm{eV})$ & $E_{p}(\mathrm{eV})$ \\
\hline RR P3BT & 0.714 & 72.4 & 1.980 & 0.183 \\
RR P3HT & 0.745 & 72.6 & 2.000 & 0.184 \\
RR P3OT & 1.01 & 86.1 & 2.010 & 0.180 \\
RR P3DDT & 2.20 & 83.2 & 2.015 & 0.183 \\
\hline \hline
\end{tabular}




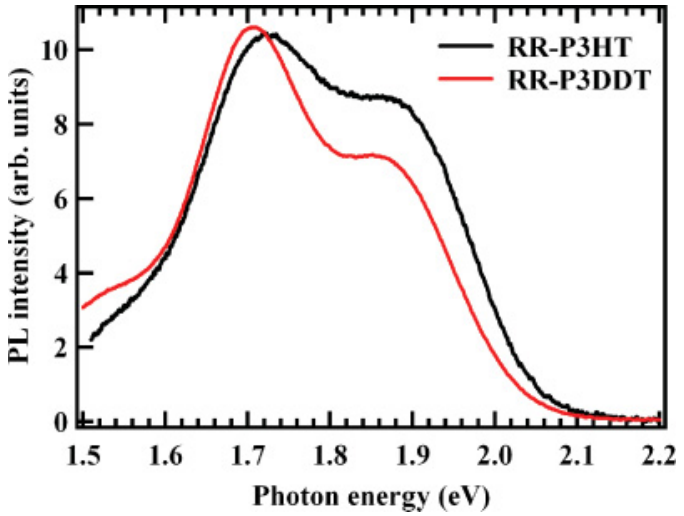

FIG. 8. (Color online) PL spectra of the RR P3HT and RR P3DDT films obtained from the cw PIA setup used in this study.

The spectra in Fig. 5 demonstrate that the bleaching spectra of the RR P3BT and RR P3HT films are given by a similar single component. It was theoretically shown that interchain couplings in the lamella aggregate component of RR P3HT cause the 0-0 transition of its PL spectrum to be forbidden unless disorder is present, ${ }^{34}$ which suggests that the 0-0 transition of the absorption spectrum can also be reduced because of interchain coupling. Therefore, the single bleaching component of RR P3BT and RR P3HT would be a quasi-intrachain species created at certain lamella aggregates. By contrast, the bleaching spectra of RR P3OT and RR P3DDT exhibit different lineshapes from those of RR P3BT and RR $\mathrm{P} 3 \mathrm{HT}$. It is thus concluded that the bleaching component of RR P3BT and RR P3HT is stabilized by the reduced interlamella distance in the P3AT films. Slight deformation of the lamella structure or weakened interchain couplings due to a reduced interlamella distance might create the bleaching component.

The difference in the bleaching line shape among the P3AT films is represented by the HR factors given in Table I. The HR factor gives a measure of the strength of the electron-phonon couplings. A trend in the obtained HR factors thus indicates that the electron-phonon couplings of final migration sites become strong with increasing interlamella distance in the P3AT film. Figure 8 shows PL spectra of the RR P3HT and RR P3DDT films obtained from the same samples as those used in the cw PIA measurements. Comparison of the two PL spectra demonstrates that the intensity ratio of the $0-1$ peak to the 0-0 peak of RR P3HT is smaller than that of RR P3DDT, which suggests that the HR factor of PL in RR P3HT is smaller. Therefore, the electron-phonon couplings of excitons responsible for PL increase with increasing interlamella distance in the P3AT film, which is consistent with the results of the bleaching spectra. This trend in PL is important, because the steady-state absorption spectra of the P3AT films are generally featureless and not largely dependent on the length of the alkyl sidechains, ${ }^{35}$ as also suggested from the EA spectra in Fig. 7. This indicates that, although the steady-state absorption is determined by major interchain aggregate components, final migration minor sites observed in the cw PIA bleaching spectra primarily determine the photophysical properties of P3AT films under $\mathrm{cw}$ photoexcitation, as seen in steady-state PL spectra.

\section{CONCLUSIONS}

The spectral features of excitons, photocarriers, and device carriers in RR P3AT films were investigated based on cwand fs-transient PIA and DM techniques. Comparison of the cw PIA and DM spectra of the P3HT film revealed that photocarriers generated by $\mathrm{cw}$ photoexcitation are dominated by localized polarons, whereas charge injection primarily creates delocalized polarons. The photoexcitation intensity dependence of the cw PIA signals demonstrated that both localized and delocalized polarons generated by photoexcitation obey a bimolecular recombination process under $\mathrm{cw}$ photoexcitation. The DM technique revealed that both DP and LP components rapidly increase at a rise point of the bias current. The proportion of positive or negative polarons in the photoexcitations was estimated by evaluating the intensity ratio of bleaching and absorption signals in the DM and cw PIA spectra to be $1 /\left(1+0.137 I^{1 / 2}\right)$ for the photoexcitation intensity $I\left(\mathrm{~mW} / \mathrm{cm}^{2}\right)$.

The final migration sites of photoexcited excitons in the P3AT films were investigated from the $\mathrm{cw}$ PIA bleaching spectra. It was demonstrated that the line shape of bleaching spectra changes with the length of the alkyl sidechains of P3AT. FC analyses showed that the cw PIA bleaching spectra of the P3AT films were almost reproduced by a simple FC progression using a single HR factor. The analyses revealed a trend that the electron-phonon couplings of final migration sites become strong with increasing interlamella distance in P3AT films. This indicates that interlamella couplings affect the electron-phonon couplings of the quasi-intrachain species created at two-dimensional lamellas.

\section{ACKNOWLEDGMENTS}

This work was supported in part by a Grant-in-Aid (No. 10016820) from the Japanese Ministry of Education, Culture, Sports, Science, and Technology. K.K. acknowledges a fund from the CASIO Science Promotion Foundation. H.H. would like to thank the Nissan Foundation and HFSP for financial support.

\footnotetext{
*Corresponding author: kkane@sci.osaka-cu.ac.jp

${ }^{1}$ R. D. McCullough, S. T.-Nagle, S. P. Williams, R. D. Lowe, and M. Jayaraman, J. Am. Chem. Soc. 115, 4910 (1993).

${ }^{2}$ T. A. Chen, X. M. Wu, and R. D. Rieke, J. Am. Chem. Soc. 117, 233 (1995).

${ }^{3}$ H. Sirringhaus, P. J. Brown, R. H. Friend, M. M. Nielsen,
}

K. Bechgaard, B. M. W. Langeveld-Voss, A. J. H. Spiering, R. A. J. Janssen, E. W. Meijer, P. Herwig, and D. M. de Leeuw, Nature (London) 401, 685 (1999).

${ }^{4}$ O. J. Korovyanko, R. Österbacka, X. M. Jiang, Z. V. Vardeny, and R. A. J. Janssen, Phys. Rev. B 64, 235122 (2001). 
${ }^{5}$ C. -X. Sheng, M. Tong, S. Singh, and Z. V. Vardeny, Phys. Rev. B 75, 085206 (2007).

${ }^{6}$ I. -W. Hwang, D. Moses, and A. J. Heeger, J. Phys. Chem. C 112, 4350 (2008).

${ }^{7}$ S. Cook, A. Furube, and R. Katoh, Energy Environ. Sci. 1, 294 (2008).

${ }^{8}$ J. Piris, T. E. Dykstra, A. A. Bakulin, P. H. M. van Loosdrecht, W. Knulst, M. T. Trinh, J. M. Schins, and L. D.A. Siebbeles, J. Phys. Chem. C 113, 14500 (2009).

${ }^{9}$ K. Kanemoto, M. Yasui, D. Kosumi, A. Ogata, M. Sugisaki, T. Karasawa, I. Akai, and H. Hashimoto, Phys. Rev. Lett. 103, 187402 (2009).

${ }^{10}$ J. Guo, H. Ohkita, H. Benten, and S. Ito, J. Am. Chem. Soc. 131, 16869 (2009).

${ }^{11}$ R. A. Marsh, J. M. Hodgkiss, S. Albert-Seifried, and R. H. Friend, Nano Lett. 10, 923 (2010).

${ }^{12}$ K. Meng, Q. Ding, S. Wang, Y. He, Y. Li, and Q. Gong, J. Phys. Chem. B 114, 2602 (2010).

${ }^{13}$ C. Botta, D. D. C. Bradley, R. H. Friend, and A. Musco, Synth. Met. 55-57, 206 (1993).

${ }^{14}$ R. Österbacka, C. P. Au, X. M. Jiang, and Z. V. Vardeny, Science 287, 839 (2000).

${ }^{15}$ M. Westerling, R. Österbacka, and H. Stubb, Phys. Rev. B 66, 165220 (2002).

${ }^{16}$ S. Günes, H. Neugebauer, and N. S. Sariciftci, Chem. Rev. 107, 1324 (2007).

${ }^{17}$ B. C. Thompson and J. M. J. Fréchet, Angew. Chem. Int. Ed. 47, 58 (2008).

${ }^{18}$ N. F. Colaneri, D. D. C. Bradley, R. H. Friend, P. L. Burn, A. B. Holmes, and C. W. Spangler, Phys. Rev. B 42, 11670 (1990).

${ }^{19}$ C. Botta, S. Luzzati, R. Tubino, D. D. C. Bradley, and R. H. Friend, Phys. Rev. B 48, 14809 (1993).

${ }^{20}$ M. Wohlgenannt, W. Graupner, G. Leising, and Z. V. Vardeny, Phys. Rev. B 60, 5321 (1999).
${ }^{21}$ M. Wohlgenannt and Z. V. Vardeny, Synth. Met. 125, 55 (2002).

${ }^{22}$ M. Westerling, C. Vijila, R. Österbacka, and H. Stubb, Chem. Phys. 286, 315 (2003).

${ }^{23}$ S. M. Lipson, J. N. Coleman, A. Drury, D. F. O’Brien, W. J. Blau, A. J. Cadby, P. A. Lane, and D. D. C. Bradley, J. Appl. Phys. 95, 6138 (2004).

${ }^{24}$ A. S. Dhoot, D. S. Ginger, D. Beljonne, Z. Shuai, and N. C. Greenham, Chem. Phys. Lett. 360, 195 (2002).

${ }^{25}$ P. A. Lane, J. C. deMello, R. B. Fletcher, and M. Bernius, Appl. Phys. Lett. 83, 3611 (2003).

${ }^{26}$ K. Kanemoto, A. Ogata, N. Inoue, T. Kusumoto, H. Hashimoto, I. Akai, and T. Karasawa, Appl. Phys. Lett. 97, 033307 (2010).

${ }^{27}$ D. Polli, M. R. Antognazza, D. Brida, G. Lanzani, G. Cerullo, and S. De Silvestri, Chem. Phys. 350, 45 (2008).

${ }^{28}$ D. Kosumi, T. Kusumoto, R. Fujii, M. Sugisaki, Y. Iinuma, N. Oka, Y. Takaesu, T. Taira, M. Iha, H. A. Frank, and H. Hashimoto, Chem. Phys. Lett. 483, 95 (2009).

${ }^{29}$ D. Kosumi, K. Abe, H. Karasawa, M. Fujiwara, R. J. Cogdell, H. Hashimoto, and M. Yoshizawa, Chem. Phys. 373, 33 (2010).

${ }^{30} \mathrm{~W}$. Helfrich, in Physics and Chemistry of the Organic Solid State, edited by D. Fox, M. M. Labes, and A. Weissberger (Interscience, New York, 1967), p. 1.

${ }^{31}$ F. Alkan and U. Salzner, J. Phys. Chem. A 112, 6053 (2008).

${ }^{32}$ M. Liess, Z. V. Vardeny, and P. A. Lane, Phys. Rev. B 59, 11053 (1999).

${ }^{33}$ H. Kishida, K. Hirota, T. Wakabayashi, B.-L. Lee, H. Kokubo, T. Yamamoto, and H. Okamoto, Phys. Rev. B 70, 115205 (2004).

${ }^{34}$ F. C. Spano, J. Chem. Phys. 122, 234701 (2005); 126, 159901 (2007).

${ }^{35}$ R. D. McCullough, R. D. Lowe, M. Jayaraman, and D. L. Anderson, J. Org. Chem. 58, 904 (1993). 